\title{
Injection into the LNLS UVX Electron Storage Ring
}

\author{
Liu Lin \\ LNLS - Laboratório Nacional de Luz Síncrotron \\ Cx. Postal 6192 - Campinas - SP - Brazil
}

\section{Abstract}

To inject the $1.15 \mathrm{GeV}$ electron storage ring - UVX - a bcam from a linear accelerator - MAIRA - is used. The electrons are injected and accumulated at low energy (100MeV) until the nominal current of $100 \mathrm{~mA}$ is reached and then are ramped to the nominal energy. A study on a conventional injection scheme has been carried out. Two injection modes are investigated: injection with the phase ellipse parameters matched and mismatched to the ring's acceptance. The mismatched mode is optimized to fit the maximum of the injected heam into the acceptance.

\section{INTRODUCTION}

To inject the $1.15 \mathrm{GeV}$ electron storage ring - UVX[1] - a beam from a linear accelerator - MAIRA[2] - is used. The electrons are injected and accumulated at low energy (100 $\mathrm{MeV}$ ) until the nominal current of $100 \mathrm{~mA}$ is reached and then are ramped to the nominal energy. The low encrgy injection presents some problems that make it more difficult than the high energy injection: short beam lifetime, long betatron oscillation damping time and enlargement of beam dimensions due to the intra-beam scattering effect (IBS); on the other hand it has the advantage of requiring a less expensive injector system. Two injection schemes are being used in this case: conventional injection and injection with anomalous repetition rate[3].

In the conventional injection, the linac pulses are injected at a rate determined by the stored beam oscillation damping time, whereas in the injection with anomalous repetition rate the pulses are injected at a much faster rate and the stored beam does not damp.

In this report a study on the conventional injection into UVX is described.

\section{INJECTION SCHEME}

The injection into the UVX storage ring takes place in one of the long straight sections, in the radial plane and from the inner side of the circumference. The electron beam from the linac is transported by an underground transfer line and is brought into the storage ring by two horizontal deflection septa. The beam is injected into the storage ring's acceptance which is locally deflected in the radial plane by three fast kickers.

The beam from the linac has the following characteristics:

- Energy $=100 \mathrm{MeV}$

- Macropulse current $=200 \mathrm{~mA}$

- Pulse length $=200 \mathrm{~ns}$

- r.m.s. emittance $=6.7 \times 10^{-7}$ rad.m. ,

and the UVX storage ring running at $100 \mathrm{MeV}$ has the following ones:

- Circumference $=77.3977 \mathrm{~m}$

- Revolution period $=258 \mathrm{~ns}$

- Harmonic number $=129$

- $\mathrm{RF}$ frequency $=500 \mathrm{MHz}$

- Horizontal betatron damping time $=16.7 \mathrm{~s}$

- Vertical betatron damping time $=16.0 \mathrm{~s}$

- Synchrotron damping time $=7.8 \mathrm{~s}$

- Equilibrium r.m.s. emittance $=1.0 \times 10^{-6}$ rad.m

The equilibrium r.m.s. emittance of the stored beam at 100 $\mathrm{MeV}$ is calculated including IBS, which is, at this energy, the dominating excitation effect. The calculations show[4] that in the range of considered values, the equilibrium emittance increases with the current per bunch and with peak RF voltage. As the greater the emittance the more critical the injection, we assume a worst case condition of $5 \mathrm{~mA}$ per bunch, $600 \mathrm{kV}$ peak RF voltage and $13 \Omega$ ring impedance with Spear scaling. These result in the value of emittance given above. The radial r.m.s. stored beam size at the injection point (end of septum) is then, $\sigma^{\mathrm{s}}{ }_{\mathrm{x}}=3.8 \mathrm{~mm}$.

At the injection point the deflected closed orbit is $15 \mathrm{~mm}$ from the central orbit and at an angle of $-0.95 \mathrm{mrad}$ with respect to it. The distance from the vacuum chamber wall corresponds to $4 \sigma_{\mathrm{x}}^{\mathrm{S}}$. The radial stability limit (dynamic aperture) assumed for the calculations is $\pm 29 \mathrm{~mm}[1] .5 \mathrm{~mm}$ are reserved for the septum wall thickness. After the injection point the deflected orbit goes through a focussing quadrupole, a defocussing quadrupole, a dipole and finally crosses the central orbit at $57 \mathrm{~cm}$ from the last element, where it is kicked again to the central orbit. The position of the elements, the bumped stored beam and the injected beam are shown in figure 1. Table I lists the kicker strengths necessary to deflect the closed orbit by the amplitude $A$ at the injection point.

Two injection modes were studied: injection with the phase ellipse parameters matched and mismatched to the ring's acceptance. The mismatched mode is optimized to fit the maximum of the injected beam into the available bumped acceptance region. Figures 2 and 3 show the injected beam and the bumped acceptance in the radial phase space at the injection point, illustrating the difference between the two injection modes.

The kickers must be turned off in 4 turns to prevent the injected beam from colliding with the vacuum chamber wall. Figure 4 shows the evolution of the injected beam, the bumped acceptance and the stored beam for the mismatched injection mode, assuming that the kicker strengths fall linearly to zero in three turns.

This process is repeated after damping of the beam with the injection of a new pulse from the linac. 


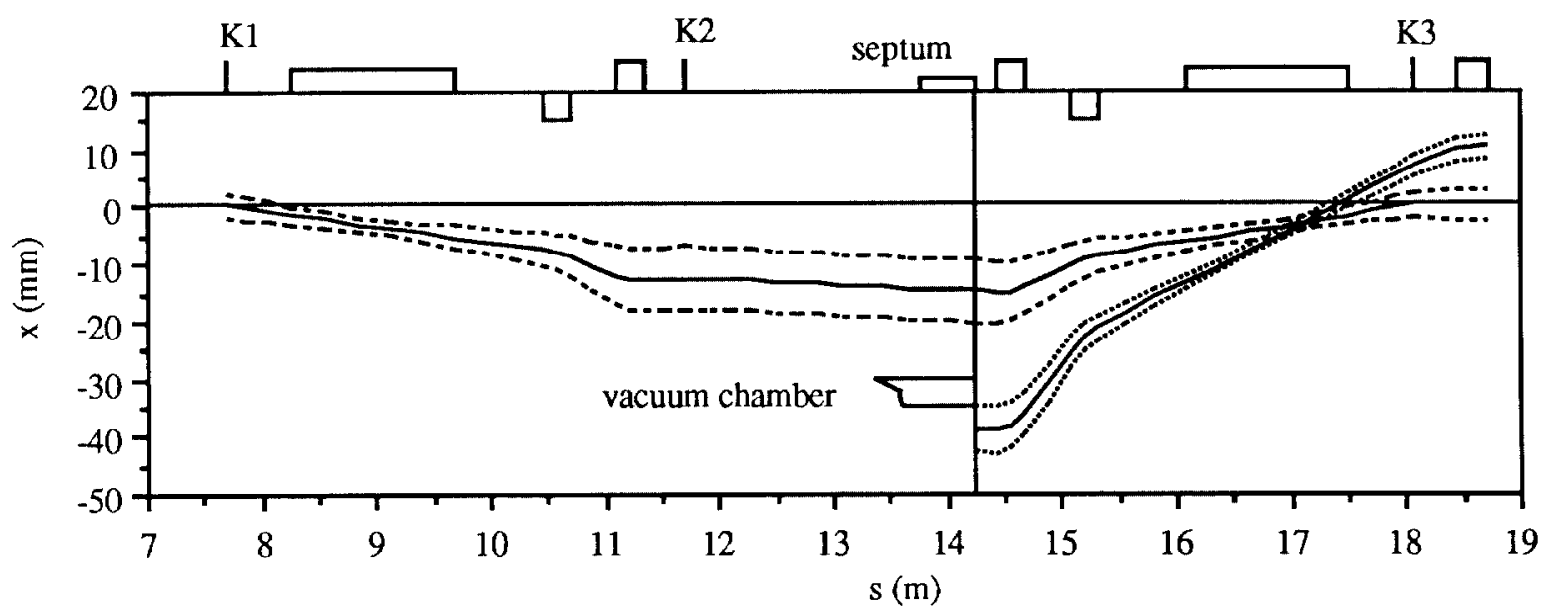

Figure 1: Schematic injection process showing the kickers position and the trajectories of the injected and stored beams. The envelope enclosing a phase space area of 2 times the r.m.s. stored beam emittance and the envelope of the injected beam are shown in dotted lines.

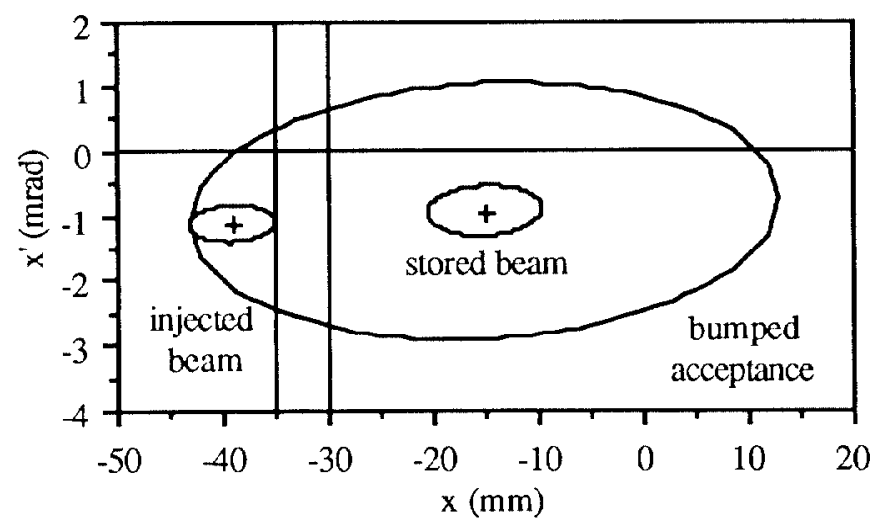

Figure 2: Bumped acceptance, injected and stored beams in the radial phase space, at the injection point (end of septum), for the matched injection mode. The area of the stored beam in the figure is two times the equilibrium emittance of the ring at $100 \mathrm{MeV}$, and that of the injected beam is 1.7 times the linac r.m.s. emittance.

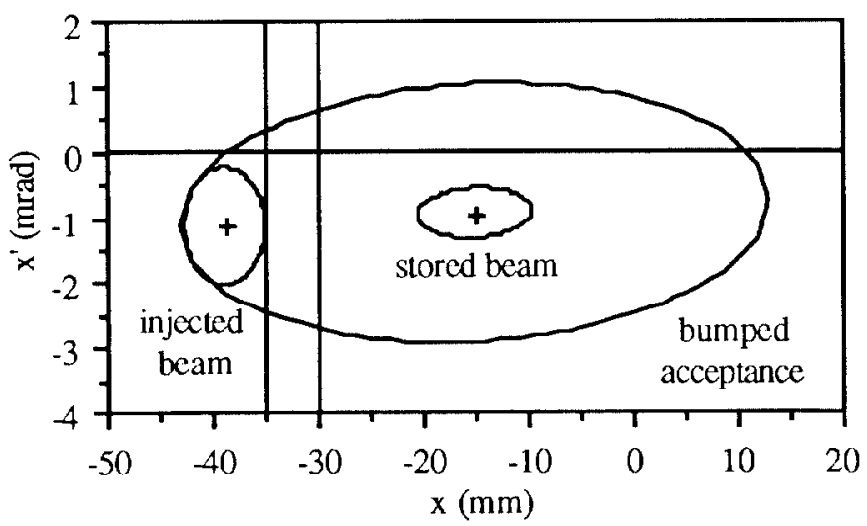

Figure 3: Bumped acceptance, injected and stored beams in the radial phase space, at the injection point (end of septum), for the mismatched injection mode. The area of the stored beam in the figure is two times the equilibrium emittance of the ring at $100 \mathrm{MeV}$, and that of the injected beam is 5.1 times the linac r.m.s. emittance.
Table I : Kicker strengths for $15 \mathrm{~mm}$ and $30 \mathrm{~mm}$ bump amplitudes at the injection point.

\begin{tabular}{lcc}
\hline & $\mathrm{A}=15 \mathrm{~mm}$ & $\mathrm{~A}=30 \mathrm{~mm}$ \\
\hline $\boldsymbol{\theta}$ kicker1 (mrad) & -2.8 & -5.6 \\
$\theta$ kicker2 (mrad) & -1.8 & -3.5 \\
$\theta$ kicker3 (mrad) & -3.3 & -6.6 \\
\hline
\end{tabular}

The parameters of the injected beam for the two injection modes are shown in table II.

Table II: Parameters of the injected beam for the two injection modes.

\begin{tabular}{lcc}
\hline & Matched injection & Mismatched injection \\
\hline $\mathrm{x}$ centroid (mm) & -39.0 & -38.8 \\
$\mathrm{X}^{\prime}$ centroid (mrad) & -1.14 & -1.14 \\
$\beta_{\mathrm{x}}(\mathrm{m})$ & 14.20 & 4.25 \\
$\alpha_{\mathrm{X}}$ & -0.096 & 0.0 \\
$\mathrm{~A}^{*}(\mathrm{rad} . \mathrm{m})$ & $1.1 \times 10^{-6}$ & $3.4 \times 10^{-6}$ \\
$\mathrm{~F}^{* *}(\%)$ & 56 & 92 \\
\hline${ }^{*} \mathrm{~A}$ is the phase ellipse area of the injected beam contained in \\
the ring's acceptance, as shown in figures 2 and 3. \\
$*_{*} \mathrm{~F}$ is the fraction of the injected beam contained in the \\
cllipse described above. Note that the fraction contained in the \\
acceptance is greater than $\mathrm{F}$, we have thus taken a conservative \\
value.
\end{tabular}

\section{INJECTION TIME}

The time needed to accumulate $100 \mathrm{~mA}$ in the storage ring in a multi-bunch mode is estimated. The linac provides a 200 $\mathrm{ns}$ and $200 \mathrm{~mA}$ pulse. Only the electrons injected into the storage ring's RF buckets are captured in a bunch. The phase trajectories of the electrons for the UVX operating at $100 \mathrm{MeV}$ with $600 \mathrm{kV}$ peak RF voltage are shown in figure 5 . The period in the graph, determined by the RF system, is 2 ns and 
the injected pulse fills 100 buckets (out of 129). The maximum amplitude of a stable oscillation of $\tau$ is $\tau_{\max }=1$ ns and the RF energy aperture is $\varepsilon_{\max }=5.4 \%$. Figure 5 also shows the energy deviation limit of $\pm 3 \%$ due to physical aperture. We observe that a small fraction of the beam is injected outside the stable region. We recall that distribution of the injected particles is gaussian in energy with $2 \%$ standard deviation (beam from the linac).
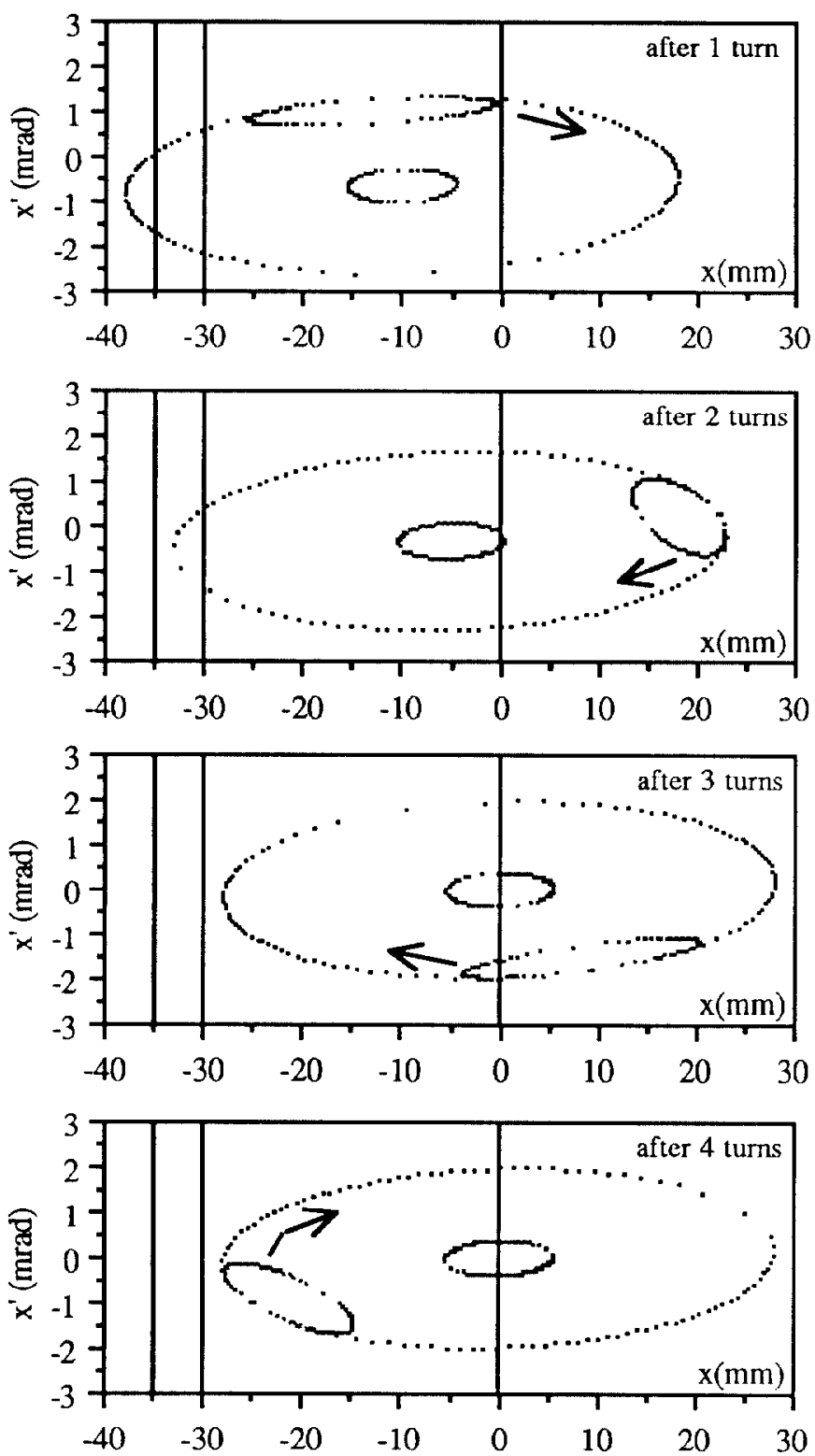

Figure 4: Evolution of the injected beam and bumped acceptance in the mismatched injection mode.

The efficiency $\eta$ of the injection process depends on the efficiency in the various stages of the process. Table III shows the calculated and supposed values in the various stages.
Table III: Efficiency at the various stages of the injection process.

\begin{tabular}{lcc}
\hline & $\begin{array}{c}\text { Matched } \\
\text { injection }\end{array}$ & $\begin{array}{c}\text { Mismatched } \\
\text { injection }\end{array}$ \\
\hline Transport line $^{[5]}\left(\eta_{\mathrm{a} 1}\right)$ & $87 \%$ & $86 \%$ \\
Inj. into acceptance $\left(\eta_{\mathrm{a} 2}\right)$ & $56 \%$ & $92 \%$ \\
Other $^{*}\left(\eta_{\mathrm{b}}\right)$ & $25 \%$ & $25 \%$ \\
$\eta^{* *}$ & $14 \%$ & $21 \%$ \\
\hline
\end{tabular}

Safety factor, fraction outside the RF buckets, losses in the ramping process, etc.

${ }^{* *} \eta=\eta_{a} \cdot \eta_{b}$, where $\eta_{a}$ is the smaller of $\eta_{a 1}$ and $\eta_{a 2}$.

The accumulated current in one linac pulse is:

$$
\mathrm{I}_{\mathrm{o}}=\eta \frac{\mathrm{T}_{\mathrm{L}}}{\mathrm{T}_{\text {ring }}} \mathrm{I}_{\mathrm{L}}
$$

where $T_{L}$ and $I_{L}$ are the linac macro-pulse length and current, respectively, and $\mathrm{T}_{\text {ring }}$ is the storage ring revolution period. For the matched injection mode $\mathrm{I}_{\mathrm{O}}=21.7 \mathrm{~mA}$ and 5 pulses from the linac are needed to store $100 \mathrm{~mA}$. Injecting the pulses at intervals equal to the betatron damping time gives a injection time of 1.4 minutes. For the mismatched injection mode $\mathrm{I}_{\mathrm{O}}=32.5 \mathrm{~mA}$ and the injection time falls to 1.0 minute. These injection times are less than the beam lifetime at 100 $\mathrm{MeV}$, which is about 20 minutes.

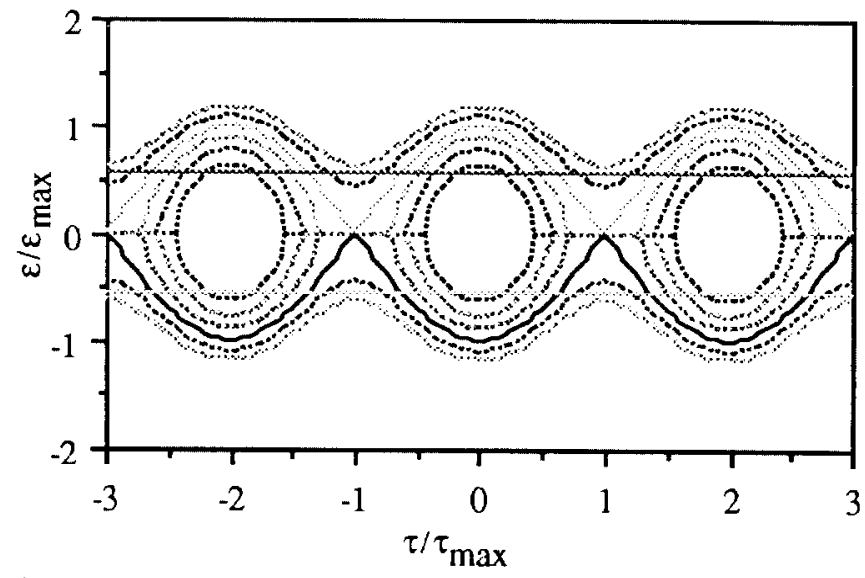

Figure 5: RF buckets for the UVX ring at $100 \mathrm{MeV}$ and with $600 \mathrm{kV}$ peak RF voltage. We have $\tau_{\max }=1 \mathrm{~ns}$ and $\varepsilon_{\max }=5.4$ $\%$. The two horizontal lines indicate $\varepsilon= \pm 3 \%$. The separatrix is shown in solid line.

\section{REFERENCES}

[1] L. Lin, L. Jahnel and P. Tavares, "A Magnet Lattice for the LNLS Soft X-Ray Source", in 1990 EPAC.

[2] A. R. D. Rodrigues and D. Wisnivesky, "Commissioning of the LNLS Injector Linac", in 1990 EPAC.

[3] E. M. Rowe, "Synchrotron Light Source Experiments: Bringing Aladdin on Line", NIM B24/25 (1987) p 414-416.

[4] "Collective Effects in the LNLS Soft X-Ray Source", LNLS internal report.

[5] Liu Lin, L. Jahnel, P. Tavares and R. H. A. Farias, "The Transport Line from MAIRA to the LNLS UVX Electron Storage Ring", these proceedings. 